\title{
SIRIS: A high resolution scanning infrared camera for examining paintings
}

\author{
David Saunders $^{* * a}$, Nick Atkinson ${ }^{\mathrm{a}}$, John Cupitt ${ }^{\mathrm{a}}$, Haida Liang ${ }^{\mathrm{b}}$, Craig Sawyers ${ }^{\mathrm{c}}$, Richard Bingham ${ }^{\mathrm{d}}$ \\ ${ }^{a}$ The National Gallery Trafalgar Square, London WC2N 5DN, UK; \\ ${ }^{\mathrm{b}}$ School of Biomedical and Natural Sciences, Nottingham Trent University, Clifton Lane, \\ Nottingham NG11 8NS, UK; \\ ${ }^{\mathrm{c}}$ Technology Enterprise, Fairway House, Haywards Road, Drayton, Oxon OX14 4LB, UK; \\ ${ }^{\mathrm{d}}$ Department of Physics and Astronomy, University College London, Gower Street, London \\ WC1E 6BT, UK
}

\begin{abstract}
The new SIRIS (Scanning InfraRed Imaging System) camera developed at the National Gallery in London allows highresolution images of paintings to be made in the near infrared region $(900-1700 \mathrm{~nm})$. Images of $5000 \times 5000$ pixels are made by moving a $320 \times 256$ pixel InGaAs array across the focal plane of the camera using two orthogonal translation stages. The great advantages of this camera over scanning infrared devices are its relative portability and that image acquisition is comparatively rapid $-\mathrm{a}$ full $5000 \times 5000$ pixel image can be made in around 20 minutes. The paper describes the development of the mechanical, optical and electronic components of the camera, including the design of a new lens. The software routines used to control image capture and to assemble the individual $320 \times 256$ pixel frames into a seamless mosaic image are also mentioned. The optics of the SIRIS camera have been designed so that the camera can operate at a range of resolutions; from around 2.5 pixels per millimetre on large paintings of up to $2000 \times 2000 \mathrm{~mm}$ to 10 pixels per millimetre on smaller paintings or details of paintings measuring $500 \times 500 \mathrm{~mm}$. The camera is primarily designed to examine underdrawings in paintings; preliminary results from test targets and paintings are presented and the quality of the images compared with those from other cameras currently used in this field.
\end{abstract}

Keywords: High-resolution, infrared, camera, examination, paintings, portable, rapid, SIRIS

\section{INTRODUCTION}

\subsection{Near infrared imaging for paintings}

Near infrared imaging techniques are used routinely in paintings conservation to examine underdrawing (the preliminary designs and sketches made by the artist before the paint is applied), to highlight changes in the composition, and to detect retouchings or overpaint. Infrared photography, using film sensitive in the spectral range 700-900 nm, has been used to examine works of art since 1930s, ${ }^{1}$ but in the late 1960s van Asperen de Boer developed infrared reflectography, which, using radiation in the 1000-2000 nm range, gives greater penetration through the surface paint layers than infrared photographic techniques, ${ }^{2}$ revealing greater detail, particularly in the underdrawing.

Throughout the 1970s and 1980s museums and galleries used infrared reflectography equipment based on infrared vidicon tubes, most notably those manufactured by Hamamatsu. To make a permanent record of the ephemeral images produced by these infrared vidicon tubes, the image was displayed on a TV monitor and a photograph of the image was made using standard black and white film. By the end of the 1980s it was quite common for the signal from the vidicon camera to be digitised, processed and stored. ${ }^{3}$ Images from vidicon cameras are, however, plagued by geometric and radiometric stability problems, so the increasing availability of solid-state cameras with sensitivity in the near infrared region has promised better quality images. Over the past few years, some museums have purchased solid-state infrared cameras, mainly based on either platinum silicide $(\mathrm{PtSi})^{4}$ or indium gallium arsenide (InGaAs) sensors, some of which are also capable of direct digital image capture.

* david.saunders@ng-london.org.uk; phone +44 2077472826

Optical Methods for Arts and Archaeology, edited by Renzo Salimbeni, Luca Pezzati, Proceedings of SPIE Vol. 5857 (SPIE, Bellingham, WA, 2005) - 0277-786X/05/\$15 - doi: 10.1117/12.612086 
However, the solid-state and vidicon infrared imaging devices share one drawback, they tend to have fairly low resolutions compared to visible region cameras. Vidicon cameras produce images at standard video resolution $(768 \times$ 576 pixels for PAL, $640 \times 480$ for NTSC), InGaAs camera can produce image sizes of up to $640 \times 480$, and PtSi devices can give images of $800 \times 512 *$. The size of image needed for a whole painting depends on the level of detail that is required - if underdrawn lines or brush strokes are to be visible, a resolution of 5 to 10 pixels per millimetre will be needed. At a resolution of five pixels per millimetre, images from the cameras described above will only cover between $120 \times 100$ and $160 \times 120 \mathrm{~mm}$ on the painting surface. These images can be stored as individual files, but if features of interest cover a larger area than a single frame, an overview of part, or the whole, of a painting is required. To achieve this, a series of images covering the surface in a grid is made and a mosaic assembly of the individual frames built. There is nothing particularly new about making image mosaics of this type - it has been common practice in astronomy and satellite mapping of the earth's surface for many years, and has been applied extensively to digitised infrared reflectogram mosaics of paintings; the procedures for image capture, correction, assembly and balancing have been refined through modifications to the standard hardware and through improved software. ${ }^{3,5-7}$

Throughout the same period, devices capable of making infrared reflectogram images of a whole painting, based on scanning an infrared-sensitive point detector over the surface of a painting have been developed in parallel. ${ }^{8}$ These have now been refined so that at each point a measurement can be made at a series of wavelengths, and the resulting visible and infrared images are not only of very high quality, but superpose exactly. ${ }^{9}$

Both the methods described above (making a grid of images using a camera and assembling a mosaic subsequently, or scanning with a point sensor) have two main disadvantages. First, making an infrared image of a painting takes many hours. Second, the equipment required to scan the point sensor or camera across the surface of the paintings is generally heavy and large. We wished to have a system that was fast, and as compact and portable as possible, and chose to build upon experience gained during the construction and deployment of a scanning colour camera at the National Gallery.

\subsection{A scanning camera}

In the early 1990s, the National Gallery participated in a project to build a high-resolution colour camera to image paintings, based on the notion of moving an area array across the focal plane of a large format lens by means of two orthogonal motorised stages. The resulting prototype camera was capable of producing images with a resolution of up to $20000 \times 20000$ pixels using a sensor with only $500 \times 290$ pixels. ${ }^{10}$ Although imaging speed is not particularly important for the application, 45 minutes were required to make a single image. A refined version of the camera based on the same principles, was subsequently developed which could produce a $10000 \times 10000$ pixel colour image in less than three minutes; this camera (the MARC II camera) is used extensively at the National Gallery (and elsewhere), and during 2000-2002 was applied to make images of the entire painting collection. ${ }^{11}$

An obvious application of the principles behind these visible region cameras was to construct an analogous camera containing a sensor that responded in the near infrared region of the spectrum.

\section{CAMERA ELEMENTS}

\subsection{Near infrared focal plane array}

The choice of sensor was determined by three principal factors, spectral sensitivity, practicability, and cost. The first consideration was the wavelength range required to reveal underdrawings. An examination of the visibility of underdrawing lines concluded that a wavelength of around $1800 \mathrm{~nm}$ gave optimal visibility, but because the types of material in the overlying layers vary, the optimum wavelength to reveal underdrawing might need to be determined empirically ${ }^{12}$. It has been claimed that lead sulphide vidicons are sensitive up to around $2000 \mathrm{~nm}$, but Walmsley et al. estimate that under normal operating conditions, the system responds to radiation up to $1600 \mathrm{~nm}$ and is most sensitive in the range from around 1000 to $1400 \mathrm{~nm} .{ }^{4}$ However, vidicon tubes are subject to the operational problems described above and focal plane sensors based on lead sulphide are not available. Furthermore, although Walmsley et al. concluded that germanium $(\mathrm{Ge})$ sensors gave good results in terms of revealing underdrawing, ${ }^{4}$ no Ge arrays were available.

\footnotetext{
* Larger custom-built infrared-sensitive arrays are available, for example those used in astronomical instruments
} 
Four solid-state sensor technologies were considered: PtSi, InGaAs, mercury cadmium telluride (MCT or HgCdTe) and indium antimonide (InSb), see Fig. 1; each has been used in astronomical applications and their properties have been widely reported. The spectral sensitivity of each detector varies with operating temperature, and several of these sensors require cooling (to as low as $c$. 80K) to obtain satisfactory signal-to-noise ratios. The need to cool a sensor would greatly have increased the potential cost of the sensor unit and made its incorporation into a scanning camera impractical, so the different materials were evaluated in terms of their spectral response and sensitivity at near ambient temperature.

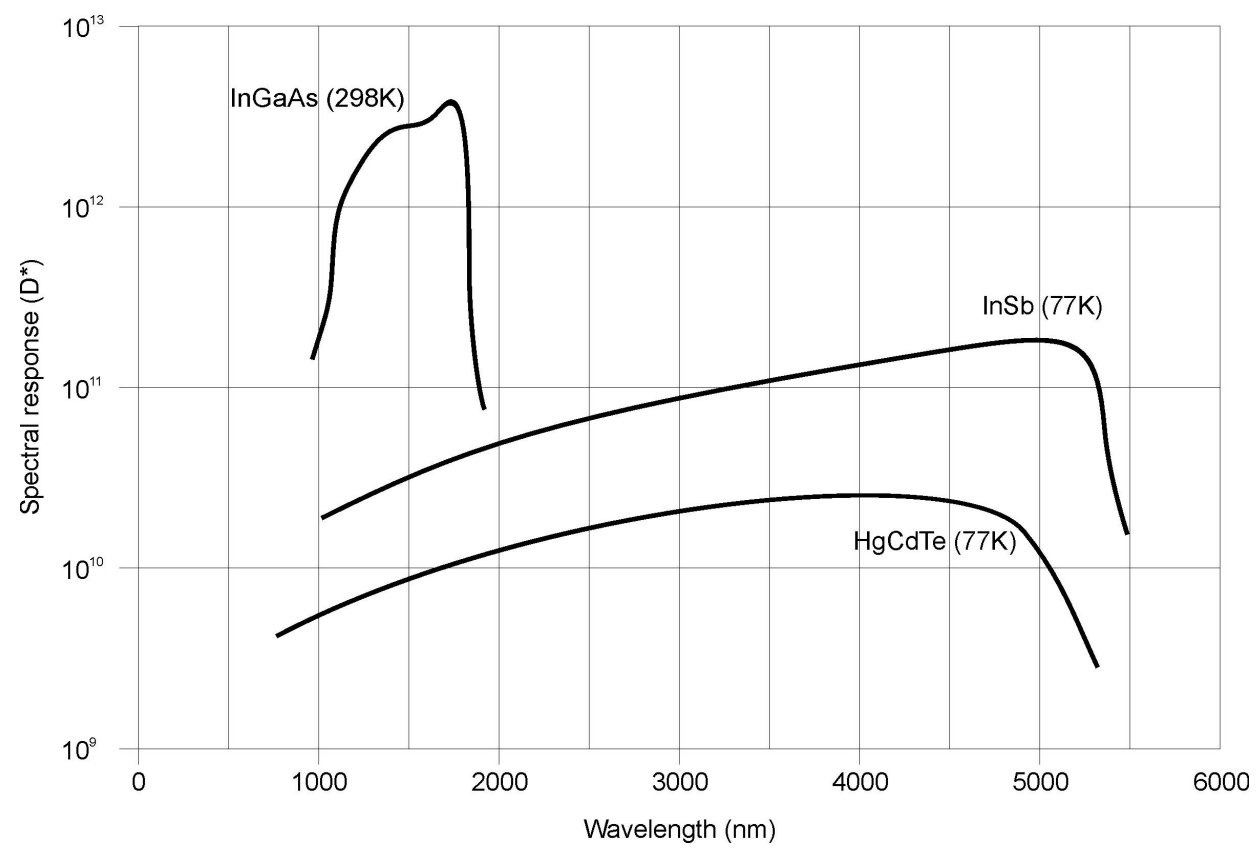

Fig. 1. Spectral response characteristics for three of the sensors considered: $\mathrm{D}^{*}$ is the detectivity in $\mathrm{cm} \cdot \mathrm{Hz}^{1 / 2} / \mathrm{W}$.

The PtSi cameras used previously to examine works of art have the dual disadvantages that they respond principally in the 3000-5000 $\mathrm{nm}$ range, having been designed as thermal imagers, and that the sensor needs to be cooled by liquid nitrogen or a closed cycle Stirling cooler. ${ }^{14,15}$ In the last few years cameras based on other sensors, notably InSb and $\mathrm{HgCdTe}$, have largely superseded PtSi devices. InSb detectors are more sensitive in the $3000-5000 \mathrm{~nm}$ region than those based on PtSi but still require cooling to operate satisfactorily. The spectral response of $\mathrm{HgCdTe}$ sensors can be tuned by adjusting the ratio of mercury to cadmium. Fig. 1 shows the response curve for a HgCdTe detector designed to operate in the $3000-5000 \mathrm{~nm}$ range, but this sensor again requires cooling before the dark current falls to an acceptable level.

InGaAs detectors have the great advantage of operating well at, or near, room temperature, giving a low dark current and good quantum efficiency $(60-70 \%)$ at $20^{\circ} \mathrm{C} .{ }^{16}$ Although the upper wavelength limit of standard InGaAs arrays is only around $1800 \mathrm{~nm}$, this should be adequate for most applications in the examination of underdrawing in traditional paintings. Based on its spectral sensitivity and ease of use, it was decided to use an InGaAs array and trials were conducted with two different InGaAs focal plane array cameras in the late 1990s.

Incorporating an InGaAs sensor into a scanning camera presented additional challenges compared to the construction of the MARC II camera. The sensor pitch for the MARC camera was $6.7 \mu \mathrm{m}$, and the fill factor low, so that by supersampling a $10000 \times 10000$ image could be made using a focal plane of $33.5 \times 33.5 \mathrm{~mm}$. However, the pixel sizes for the InGaAs arrays considered were c. $30 \mu \mathrm{m}$ and the fill factors $>90 \%$, so super-sampling was not considered. To make a $10000 \times 10000$ pixel image would have required a focal plane of $300 \times 300 \mathrm{~mm}$, which would have resulted in a camera that was considered too large to be portable; one of the aims of the project was to create a camera that could be used to make images in situ. The design was, therefore, based on creating a $5000 \times 5000$ pixel image by moving the sensor chip 
across a $150 \times 150 \mathrm{~mm}$ focal plane. Because the sensor would be moved to a number of different positions across the focal plane the array size is not critical. However, the smaller the array, the more positions are needed, since a minimum overlap between sub-images is needed to give a 'seamless' mosaic image. A compromise between size, cost and availability resulted in the selection of an Indigo Alpha $320 \times 256$ pixel camera as the basis for the scanning camera. ${ }^{17}$

\subsection{Near infrared large format lens}

The design specification for the camera called for flexibility. It was to be used to image large paintings (up to $2000 \times$ $2000 \mathrm{~mm}$ ) with a camera-to-object distance of c. $4000 \mathrm{~mm}$, but also capable of making images of smaller areas $(500 \times$ $500 \mathrm{~mm}$ ) with a camera-to-object distance of $c .1000 \mathrm{~mm}$. Assuming an image of $5000 \times 5000$ pixels in each case, the resolution range would be from 2.5 to 10 pixels per millimetre, suitable for a general inspection of a painting and a detailed study of the underdrawing respectively.

The maximum painting size and the object distance define the field-of-view that is free from vignetting, which in this case needs to be at least $39^{\circ}$. A lens with a focal length of $280 \mathrm{~mm}$ gives a magnification factor of 0.075 at an object distance of $4000 \mathrm{~mm}$, which corresponds to a resolution of 2.5 pixels per millimetre on the object plane. The diameter of the lens determines the diffraction limited resolution or Point Spread Function (PSF) of the lens, although in reality the lens PSF is usually worse than the diffraction limited resolution because of aberration. A high f-number results in a smaller lens diameter and hence reduces the mass of the lens, but the exposure time and the PSF are increased. As a compromise, a f-number of 10 was chosen, since the lens that gave satisfactory results with the Vidicon system also had a f-number of 10. A f-number of 10 gives an aperture of $28 \mathrm{~mm}$, which has a diffraction limited resolution of $c .5$ pixels per millimetre at an object distance of $4000 \mathrm{~mm}$. Hence, the combined resolution of the system is limited by the pixel size rather than the diffraction limit of the lens. However, to cope with aberration a larger diameter lens that could be stopped down to $\mathrm{f} / 10$ was designed.

While it is possible to find an off-the-shelf large format lens made for the visible range that fits these criteria, no such lenses are available to operate in the near infrared $(900-1700 \mathrm{~nm})$. This is not surprising; since off-the-shelf infrared chips are relatively small there has been no demand for large format infrared lenses. Due to chromatic aberrations, a lens designed for the visible range is unlikely to satisfy the requirements for high resolution and large field of view. In addition, because such large format lenses tend to be used in very exacting applications, every effort is made to ensure the highest quality performance possible in the visible range, and anti-reflection coatings are applied to reduce transmission in the infrared.

A lens was therefore designed specifically for this application. It is a new five-element design comprising a meniscus followed by two cemented doublets, with an internal fixed aperture stop. The design was refined after the polished glass for each element was delivered to account for small differences from the theoretical specification. An anti-reflection coating was applied that reduced the average reflection to $<1.5 \%$ in the $900-1700 \mathrm{~nm}$ region. All the elements were assembled in a tubular mount with a standard $67 \mathrm{~mm}$ diameter filter mount at the front and a custom-made plate at the rear to attach the lens to the camera body. The final lens, which had a focal length of $242 \mathrm{~mm}$, was tested and it was found that the aberration was minimal, such that the PSF of the lens was essentially diffraction limited. The error at the edge of the field under the operating conditions used is given in Table 1.

Table 1. The operating conditions used to obtain images at low, medium and high resolution, and the error at the edge of the field of view for each of these configurations

\begin{tabular}{|l|l|l|l|l|l|}
\hline $\begin{array}{l}\text { Approximate field } \\
\text { of view on object } \\
\text { plane }(\mathrm{mm})\end{array}$ & $\begin{array}{l}\text { Resolution } \\
\text { (pixels per } \\
\text { millimeter) }\end{array}$ & $\begin{array}{l}\text { Object-to-image } \\
\text { plane distance } \\
(\mathrm{mm})\end{array}$ & $\begin{array}{l}\text { Object plane-to- } \\
\text { lens distance } \\
(\mathrm{mm})\end{array}$ & $\begin{array}{l}\text { Lens-to-image } \\
\text { plane distance } \\
(\mathrm{mm}) *\end{array}$ & $\begin{array}{l}\text { Error at edge of } \\
\text { field }(\%)\end{array}$ \\
\hline $2000 \times 2000$ & 2.3 & 3991.8 & 3677.9 & 241.8 & 0.022 \\
\hline $1000 \times 1000$ & 4.6 & 2261.1 & 1927.2 & 261.7 & 0.002 \\
\hline $500 \times 500$ & 9.2 & 1427.7 & 1052.3 & 303.2 & 0.053 \\
\hline
\end{tabular}

*The lens depth from front to rear vertex is $72.2 \mathrm{~mm}$. 


\subsection{Sensor positioning system}

To translate the sensor across the focal plane, two conventional stepper-driven Unislide stages, each capable of a displacement of $150 \mathrm{~mm}$ with an accuracy of $<0.05$ per $100 \mathrm{~mm}$ were selected. Using 4000 steps per revolution the stages have an accuracy of $0.25 \mu \mathrm{m}$ and a repeatability of $<10 \mu \mathrm{m}$. To improve the repeatability the stages are always driven in the same direction when positioning the sensor prior to image capture. The InGaAs sensor unit was mounted onto the upper stage using a custom designed bracket that ensured its focal plane was parallel to the motion of the stages.

The stages are driven by high torque stepping motors, controlled individually by Xli type controllers from Parker Automation; these are linked to a computer by a serial (RS232) cable. A Hall effect type home sensor on each stage allows repositioning to a known, repeatable point. For stability the stages are mounted on the thick aluminium back plate of the camera, Fig. 2; the back plate is pocketed to reduce the overall mass of the camera while ensuring rigidity where required.

\subsection{Camera body and power supply}

The camera body needed to provide a rigid base for the translation stages holding the sensor and maintain the lens in position so that its optical axis was perpendicular to the focal plane, while allowing it to move along its focal axis by up to $c .62 \mathrm{~mm}$ to achieve the different image resolutions given in Table 1; that is to vary the lens-to-image plane distance from 241.8 to $303.2 \mathrm{~mm}$. The body also had to house the camera electronics, which were mounted to one side of the translation stages and connected to the sensor head using a very flexible 44-way ribbon cable, Fig. 2. All the internal elements of the case are anodized black to reduce unwanted reflectance.

The design called for the camera to be portable and relatively lightweight, so rigidity was achieved by attaching both the back plate with its surrounding light-tight case and the lens on a strong horizontal element, Fig. 3. In use this rigid base is mounted on an adaptor plate that fits either to an astronomical tripod or, for fieldwork and imaging in situ, to a heavyduty photographic tripod, Fig. 4 . The movement of the lens with respect to the focal plane is achieved by mounting the lens unit on an adjustable stage. The rough position of the lens is adjusted by moving a rod marked with the correct positions of three commonly used imaging resolutions, and the fine focus is then adjusted using a micrometer attached to this stage. Fig. 5 .

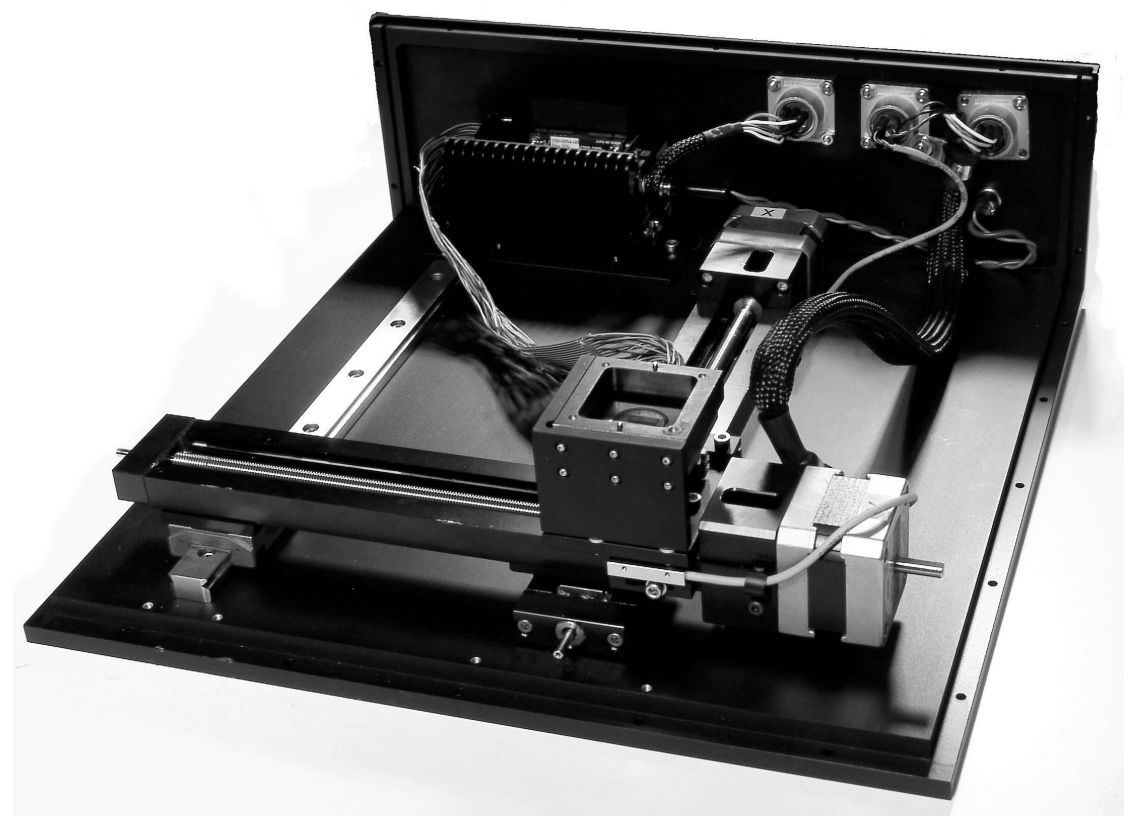

Fig. 2. The scanning stages attached to the camera back plate. The InGaAs sensor is mounted on the upper stage and connected to the camera electronics (seen at the top left) by a 44-way ribbon cable. 


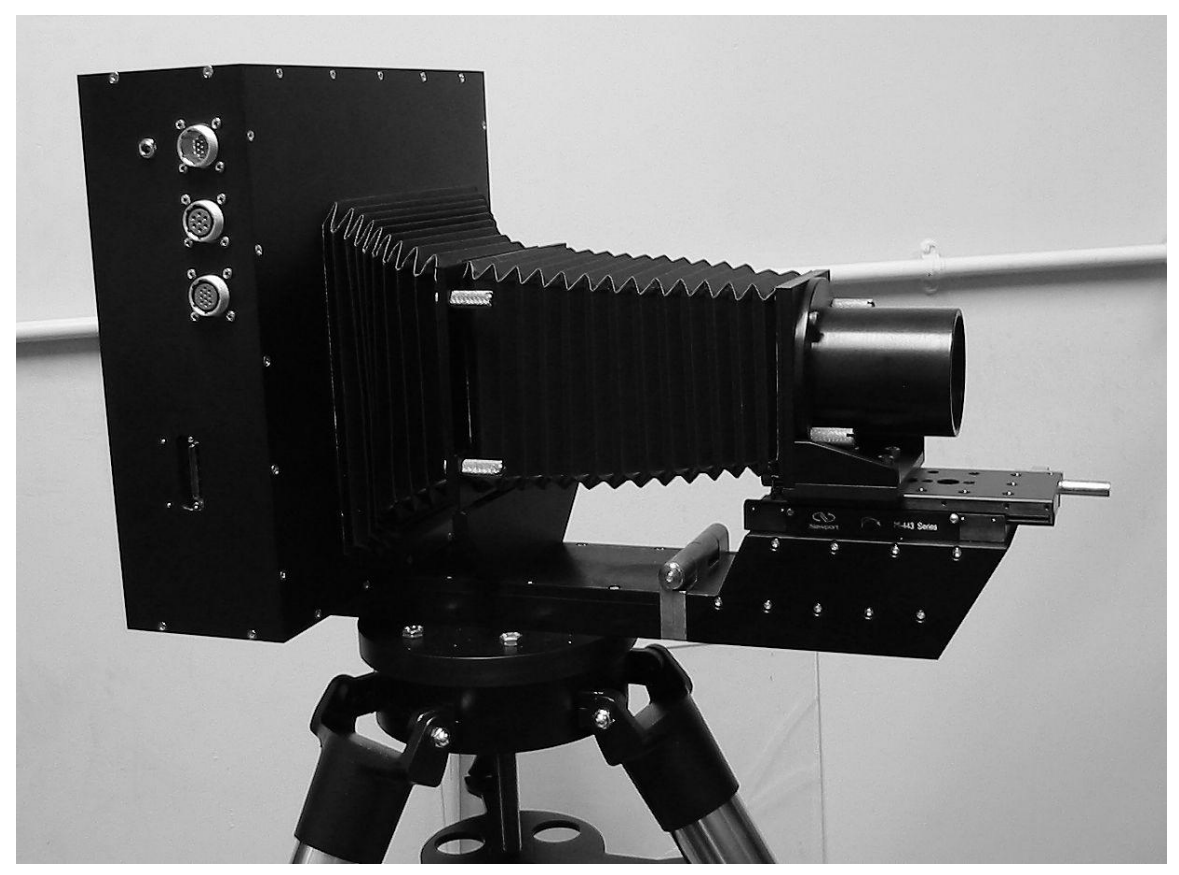

Fig. 3. The SIRIS camera from the side; the lens is mounted on an adjustable stage to the right which is in turn attached to the rigid base onto which the camera unit is also fixed. The rigid (left) and flexible (right) bellows units allow the lens to move freely and collapse for transportation.

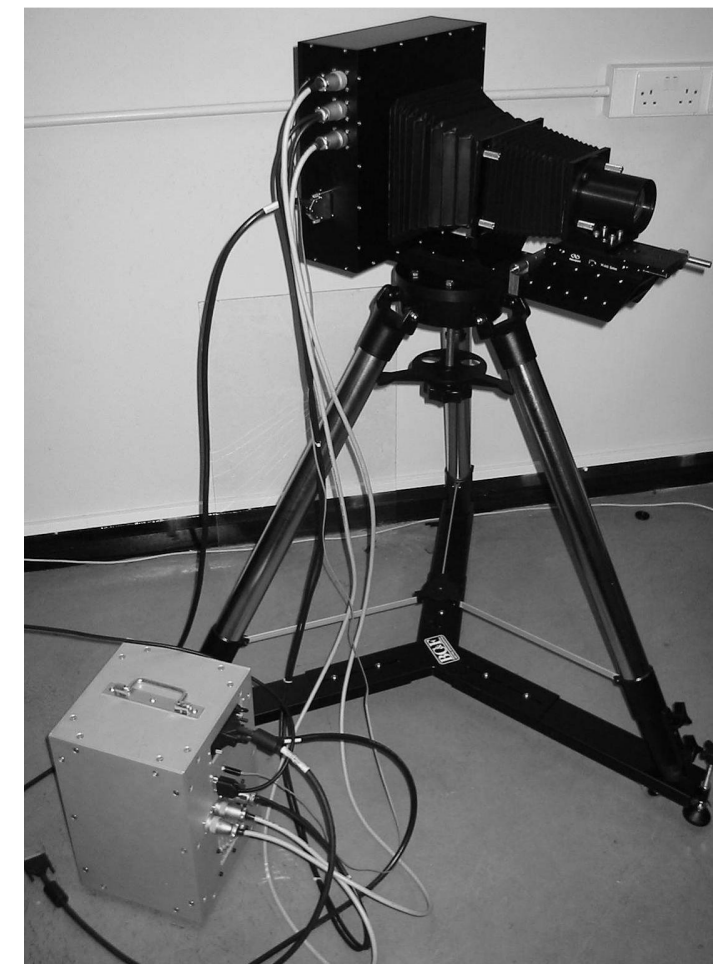

Fig. 4. The camera, tripod and control box. 


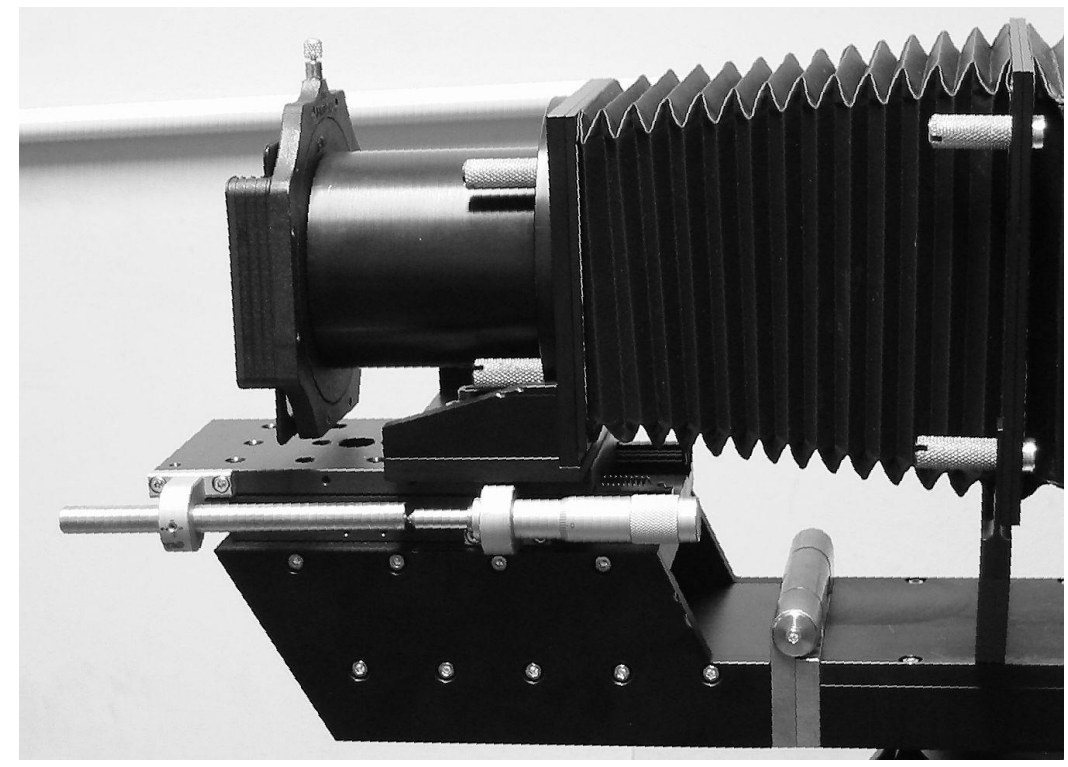

Fig. 5. The adjustable stage for the lens; the metal rod to the left allows the magnification factor to be adjusted rapidly, while the micrometer is used for fine focus.

For transportation, the lens can be removed from the stage and the rigid base, which is hinged, can be folded towards the camera to reduce its overall size, Fig. 6 . Between the lens and the case housing the stages and the camera electronics are two sets of bellows; a rigid self-supporting set nearest the case and a more flexible set nearer the lens. For transportation the bellows can be collapsed and a blanking plate attached to protect the sensor and electronics in the main camera case.

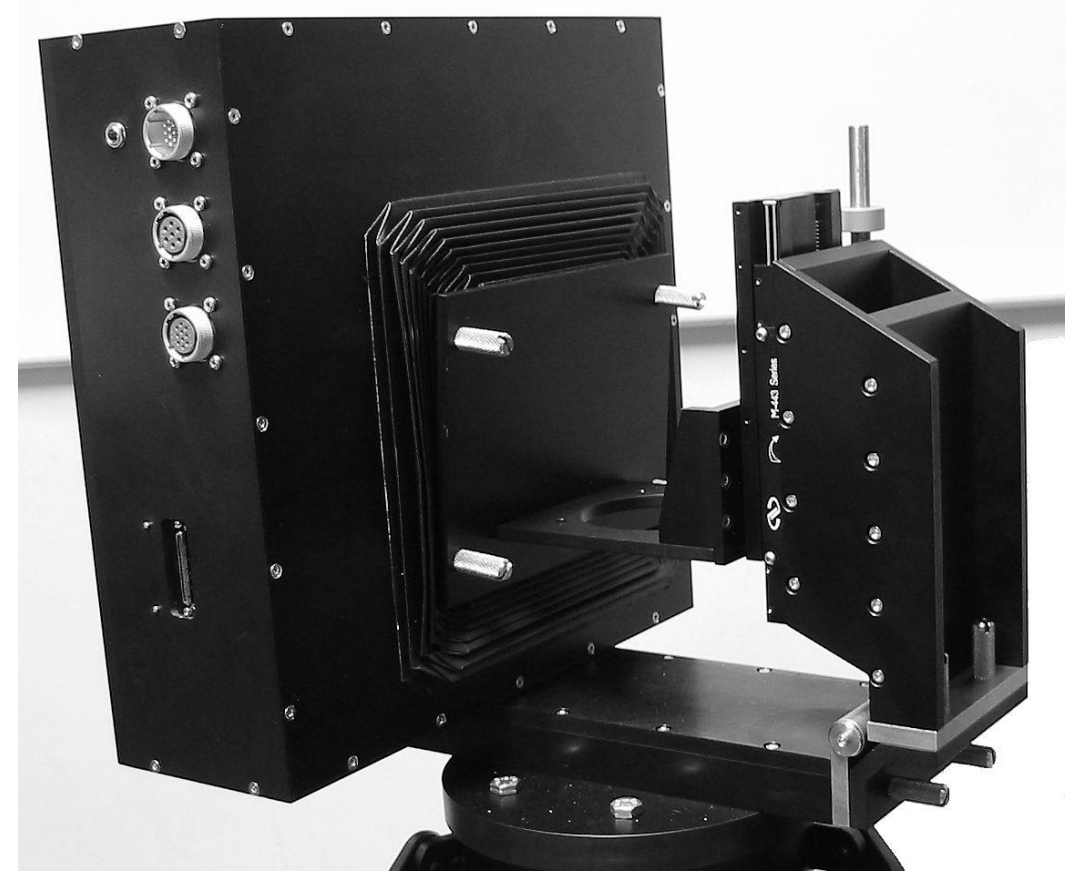

Fig. 6. The camera folded for transportation; the lens and flexible bellows have been removed and the blanking plate attached to the rigid bellows, while a bracket supports the front of the stage at a right angle to the rear. 
A box was constructed to house the motor and camera power supplies, the motor controllers, and National Instruments PCI-1422 image capture card. This control box, made from extruded aluminium section with anodised plate aluminium walls, provides all the control and power connections needed to run the camera from a single mains socket; these include a SCSI type cable connecting the image capture card to the camera electronics, power for the camera electronics, and power, control and home sensor data for each of the stages. These connections are unique, so that it should not be possible to connect the camera to the control box incorrectly. Inside the box, the image capture card is connected to a PCI to PCMCIA adapter, so that the images can be read through the PCMCIA port on a laptop computer. A serial link from the laptop to the control box is used to control the motion of the stages.

\subsection{Control and capture and calibration software}

The software to control the camera was modified from the application that was built by the Scientific Department at the National Gallery for the MARC II camera. ${ }^{7}$ The main difference was in the number of images to be made - with an image overlap of 60 pixels, a grid of $18 \times 24$ images, each of $320 \times 256$ pixels was needed to give a final image resolution of $4740 \times 4764$ pixels. A complete capture and assembly of all 432 tiles takes around 20 minutes using the current software, but this might be improved slightly by future refinement.

The SIRIS software uses the National Instruments NI-IMAQ software to grab frames from the chip. The stepper motors are driven over a serial port by a small module written in $\mathrm{C}$. The rest of the software, including the interface components to view, select, capture and save areas of the focal plane, was adapted from software written at the National Gallery for the MARC II camera.

The sensor has a high dark current (partly due, probably, to the extended cabling between the sensor and the camera electronics); this dark current also varies between the two readout circuits on the chip. Indeed, the dark current for the odd columns has the bias set at below the zero of the AD converter. To remove this additive component the software uses a dark current image of an evenly lit uniform white target taken while the camera is defocused. Neutral density filters are used to reduce the signal to the point where the leakage for both odd and even columns is just greater than zero; 32 frames are then captured and averaged. If this average image was simply subtracted from every frame it would tend to clip the signal at zero in dark parts of the image, so an additional constant positive offset of the dark frame average plus 128 is added.

To remove variations in pixel sensitivity, the software uses a set of images of a uniform white target with high illumination and dark current removed. For each pixel in each tile a scaling factor is calculated to normalise the sensitivity of that pixel relative to the brightest part of the white reference image. This correction also removes two other factors: the variation of sensitivity with the angle of illumination as the sensor travels across the focal plane, and lens vignetting.

As well as rather large variability in dark current and sensitivity, the sensor also has a number of 'dead' pixels, where dead is defined as a pixel whose value at some point in the illumination range differs from the average value by more than a number of standard deviations. The software uses a map of the dead pixels (about 34 for the sensor in the SIRIS camera) to replace each dead pixel with the median value of the 8-way connected surrounding non-dead pixels.

When the tiles are blended together to make the final image, the software analyses the overlap areas for features and displaces each tile by a small amount to reduce mosaic artifacts. This removes errors caused by, for example, sagging of the easel over the exposure period. At the same time the relative brightness of the overlap areas is checked and tiles adjusted in brightness to remove any visible tile boundaries caused by changes in lighting intensity.

\section{PRELIMINARY RESULTS}

Once the elements of the camera had been assembled and tested, the camera was used to image a test panel kept in the Scientific Department at the National Gallery. This comprises a white ground, with underdrawing in a number of typical materials, including black ink, charcoal and coloured chalks. Each type of material is covered with one or more paint layers containing traditional pigment mixtures representative of the paintings in which underdrawing is usually detected. 
This test chart had already been imaged using a Hamamatsu vidicon camera and had been examined with InGaAs and PtSi cameras during their evaluation in the 1990s. Fig. 7 compares the image made with the SIRIS camera (right image) with that made with the vidicon camera. The penetration of the paint layers is quite similar using both devices but, although the resolution in pixels per millimetre on the object is the same in both images, that made with the solid-state camera is much sharper, allowing individual underdrawing lines to be seen clearly. Fig. 8 shows comparable images for one patch on the chart; an underdrawing in black ink over the white ground has been covered with an oil paint containing the pigment lead-tin yellow. In the image form the SIRIS camera, more detail can be seen, showing the 'pooling' of the ink within the brush strokes, the result of poor wetting during its application.

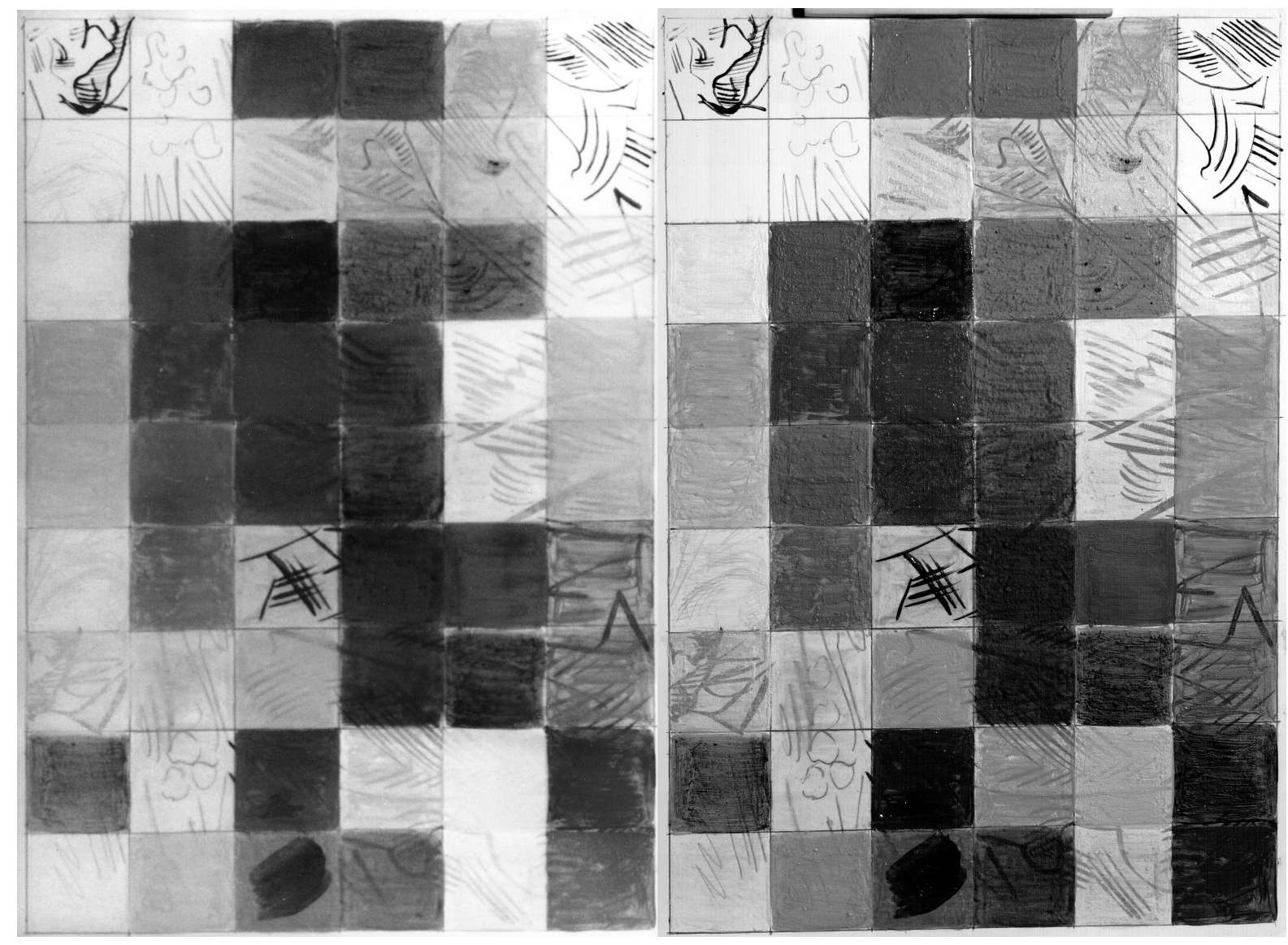

Fig. 7. Image of the underdrawing test chart: left, a mosaic assembly of reflectograms made with a Hamamatsu vidicon camera; right, an image from the SIRIS camera.
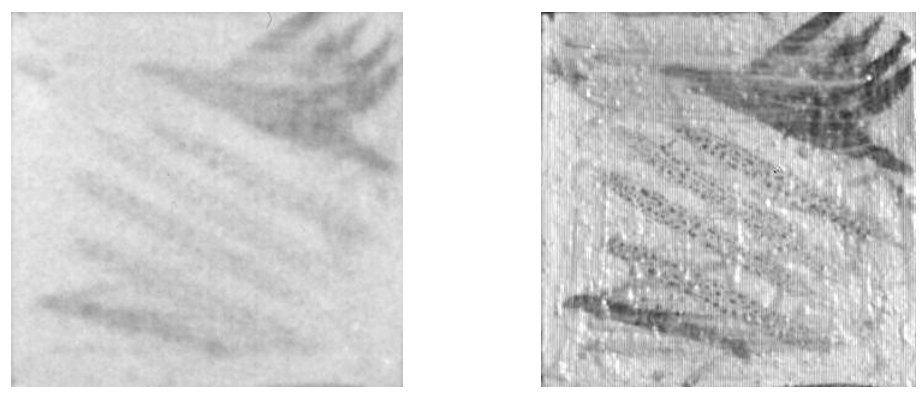

Fig. 8. One patch from the underdrawing test chart (lead-tin yellow paint over a black ink underdrawing): left, image from a Hamamatsu vidicon camera; right, image from the SIRIS camera. 
The camera was next used to image an early Netherlandish painting in the National Gallery that is known to have good underdrawing (and has frequently been used as a test painting when new infrared imaging technologies have been evaluated). Fig. 9 again compares the image made with the SIRIS camera (right image) with that made with the vidicon camera. Broadly similar penetration of the paint layers is seen using both devices but again, although the resolution in pixels per millimetre on the painting is the same, the image made by the SIRIS camera is much sharper. In addition, the sophisticated assembly procedure integrated into the capture and calibration software produces an image in which the joins between individual $320 \times 256$ pixel sub-images are not visible. In contrast some lightness differences between adjacent sub-images are visible in the image assembled from frames from the vidicon camera, particularly along the top edge of the image. However, the main advantage of the new system is its speed. An experienced operator took half a day to acquire and store the individual images from the vidicon camera and a further two working days to calibrate, correct and assemble the images into a mosaic. In contrast, after the camera and lights had been set up, the $c .5000 \times$ 5000 pixel SIRIS image was acquired, corrected and assembled in slightly under 20 minutes without further operator intervention. These timings compare favourably with point scanning infrared systems, which would take several hours to scan an equivalent area and cannot scan large paintings in a single operation.

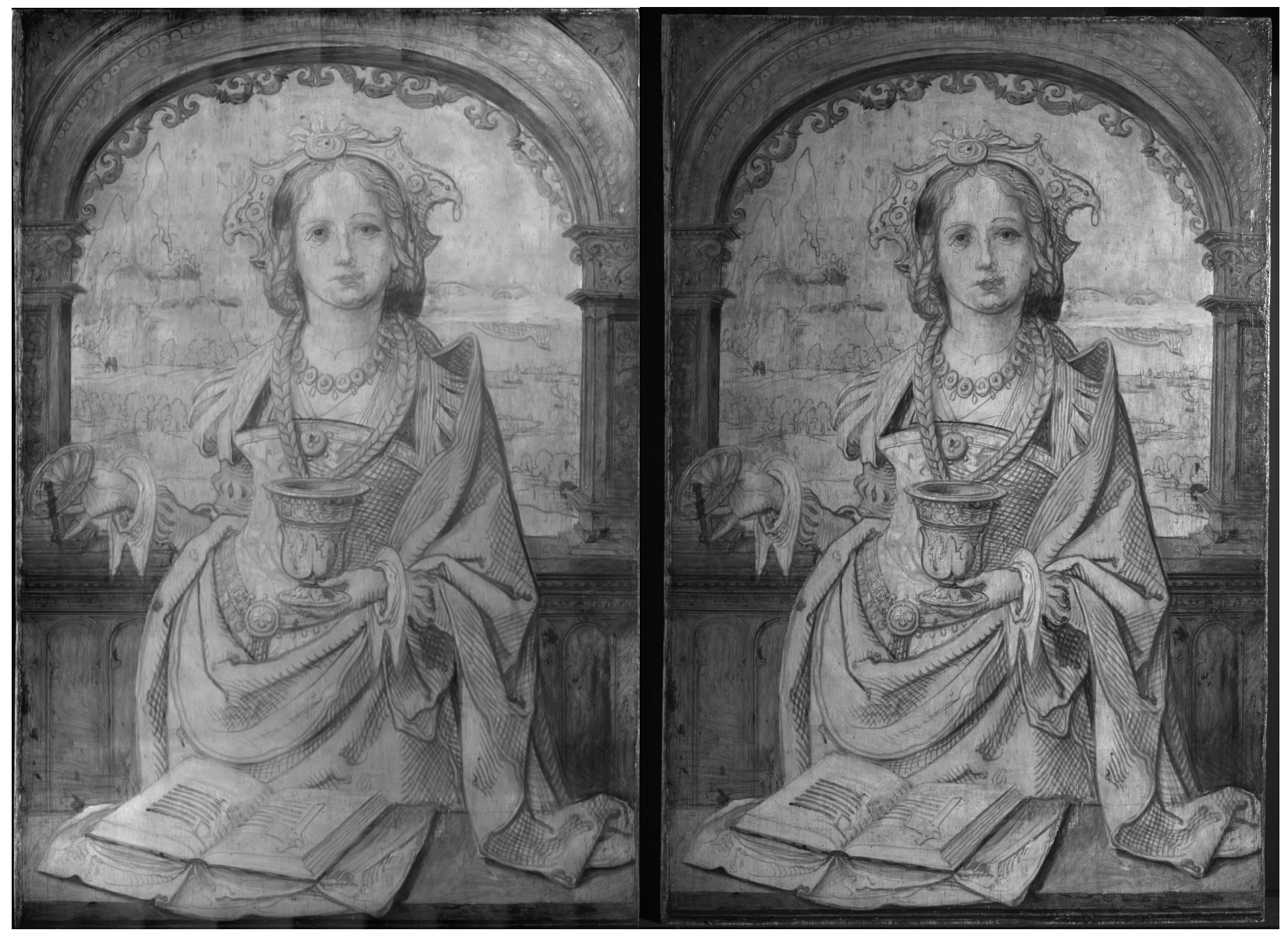

Fig. 9. The Magdalen, by an anonymous Netherlandish artist (National Gallery No. 719): left, a mosaic assembly of reflectograms made with a Hamamatsu vidicon camera; right, an image from the SIRIS camera.

\section{CONCLUSIONS AND FUTURE DEVELOPMENT}

The SIRIS camera developed at the National Gallery in London uses a well-established technology (the InGaAs sensor) in a novel application, scanning a small $(320 \times 256$ pixel array $)$ across the focal plane of a specially designed infrared lens. The resulting high-resolution images of paintings clearly reveal the underdrawing and give sharper, more 
homogeneous images than are produced by current infrared reflectographic devices. The camera has the dual advantages of speed - a full $5000 \times 5000$ pixel image can be made in around 20 minutes - and portability; the camera, control box, tripod and laptop computer can be moved relatively easily. At the time of writing, the camera is being used in the National Gallery to image paintings in situ. The camera has been designed to 'fold', so that in a suitable case it could be transported by air as hand luggage, while the control box is rugged enough to travel as cargo. If the tripod is not transported, the tripod adaptor allows the camera to be used wherever a suitable photographic tripod is available.

The thread at the front of the lens has been used to attach a $100 \times 100 \mathrm{~mm}$ filter holder. At present this contains a Kodak 87 filter to block visible light from entering the camera, but it is intended to investigate the use of other filters in due course.

The speed of operation makes the camera ideally suited for preliminary investigations in situ, where it is often necessary to examine the entire surface of a large number of paintings to determine whether any underdrawing is present and highlight those areas worthy of in-depth investigation. The camera can then be used in a higher-resolution mode to make these more detailed studies, either in situ, or in the studio. The assembly of infrared images into mosaics has often consumed the time of those interested in the conservation and art-historical implications of the underdrawing technique

or design. The SIRIS camera makes the acquisition of high-quality images a simpler, faster process, freeing time for the more intriguing task of interpreting the underdrawing.

\section{REFERENCES}

1. F.I.G. Rawlins, 'Beneath the surface of old pictures', Journal of the Royal Society of Arts 41 (1943) 150-154.

2. J.R.J van Asperen de Boer, 'Reflectography of paintings using an infra-red vidicon television system', Studies in Conservation 14 (1969) 96-118.

3. G. Wecksung, R. Evans, J. Walker, M. Ainsworth, J. Brealey and G. Carriveau, 'Assembly of infra-red reflectograms by digital processing using a portable data collecting system', in ICOM Committee for Conservation, 8th Triennial Meeting, Sydney (1987) 107-109.

4. E. Walmsley, C. Fletcher and J. Delaney, 'Evaluation of system performance of near infrared imaging devices', Studies in Conservation 37 (1992) 120-131.

5. R. Billinge, J. Cupitt, N. Dessipris and D. Saunders, 'A note on an improved procedure for the rapid assembly of infrared reflectogram mosaics', Studies in Conservation 38 (1993) 92-98.

6. A. Burmester and F. Bayerer, 'Towards improved infrared-reflectograms', Studies in Conservation 38 (1993) 145154.

7. D. Saunders, A. Burmester, J. Cupitt and L. Raffelt, 'Recent applications of digital imaging in painting conservation: transportation, colour change and infrared reflectographic studies', Tradition and Innovation: Advances in Conservation, ed. A. Roy and P. Smith, IIC, London (2000) 170-176.

8. D. Bertani, M. Cetica, P. Poggi, G. Puccioni, E. Buzzegoli, D. Kunzelman and S. Cecchi, 'A scanning device for infrared reflectography', Studies in Conservation 35 (1990) 113-116.

9. L. Pezzati, M. Materazzi and P. Poggi, 'IR-colour scanning reflectography', in The Painting Technique of Pieretro Vannucci, called il Perugino, ed. B. Brunetti, C. Seccaroni and A. Sgamellotti, Nardini Editore, Florence (2004) $65-70$.

10. R. Lenz, R. Beutelhauser and U. Lenz, 'A microscan/macroscan $3 \times 12$ bit digital color CCD camera with programmable resolution up to 20,992 × 20,480 picture elements', Proceedings of the Commission V Symposium: Close range techniques and machine vision, Melbourne, Australia, International Archives of Photogrammetry and Remote Sensing 30(5) (1994) 225-230.

11. D. Saunders, J. Cupitt, C. White, and S. Holt, 'The MARC II camera and the scanning initiative at the National Gallery', National Gallery Technical Bulletin 23 (2002) 76-82.

12. J. Delaney, C. Metzger, E. Walmsley and C. Fletcher, 'Examination of the Visibility of Underdrawing Lines as a Function of Wavelength', in ICOM Committee for Conservation, 10th Triennial Meeting, Washington DC (1993) $15-19$.

13. D. Bertani, 'La riflettografia infrarossa', in Oltre il visiblile: indagini riflettografiche, Univsersità degli studi di Milano, Milan (2001) 3-18. 
14. E. Walmsley, C. Metzger, C. Fletcher and J. Delaney 'Evaluation of Platinum Silicide Cameras for Use in Infrared Reflectography', in ICOM Committee for Conservation, 10th Triennial Meeting, Washington DC (1993) 57-62.

15. J. Coddington and S. Siano, 'Infrared Imaging of Twentieth-Century Works of Art', in Tradition and Innovation: Advances in Conservation, Contributions to the IIC Melbourne Congress, 10-14 October 2000, ed. A. Roy and P. Smith, International Institute for Conservation, London (2000) 39-44.

16. A. Manissadjian, P. Tribolet, P. Chorier and P. Fillon, 'Short-Wave InfraRed detector trade-offs and applications', Proceedings of the SPIE: Infrared Technology and Applications XXVIII 4820 (2003) 446-452.

17. http://www.indigosystems.com/product/alphanir.html 